\title{
Associated factors for recommending HBV vaccination to children among Georgian health care workers
}

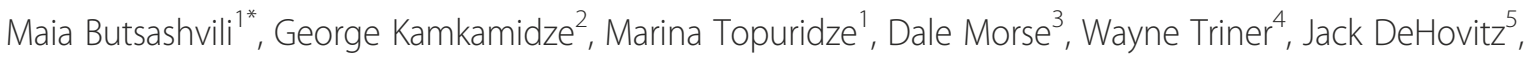 \\ Kenrad Nelson ${ }^{6}$ and Louise-Anne McNutt ${ }^{7}$
}

\begin{abstract}
Background: Most cases of hepatitis B virus (HBV) infection and subsequent liver diseases can be prevented with universal newborn HBV vaccination. The attitudes of health care workers about HBV vaccination and their willingness to recommend vaccine have been shown to impact HBV vaccination coverage and the prevention of vertical transmission of HBV. The purpose of this study was to ascertain the factors associated with health care worker recommendations regarding newborn HBV vaccination.

Methods: A cross-sectional study of prevalence and awareness of hepatitis B and hepatitis B vaccine was conducted among randomly selected physicians and nurses employed in seven hospitals in Georgia in 2006 and 2007. Self-administered questionnaires included a module on recommendations for HBV, HCV and HIV.

Results: Of the 1328 participants included in this analysis, 36\% reported recommending against hepatitis B vaccination for children, including $33 \%$ of paediatricians. Among the $70.6 \%$ who provided a reason for not recommending HBV vaccine, the most common concern was an adverse vaccine event. Unvaccinated physicians and nurses were more likely to recommend against HBV vaccine (40.4\% vs $11.4 \%$, PR 3.54; 95\% Cl: 2.38, 5.29). Additionally, health care worker age was inversely correlated with recommendations for HBV vaccine with older workers less likely to recommend it.
\end{abstract}

Conclusion: Vaccinating health care workers against HBV may provide a dual benefit by boosting occupational safety as well as strengthening universal coverage programs for newborns.

Keywords: Hepatitis B, Vaccine, Safety, Health Care Worker, Newborns

\section{Background}

Hepatitis B virus (HBV) infection remains a major global public health concern. Georgia is among the countries with an intermediate prevalence of chronic HBV infection $(2.7 \%)$, similar to that of other Eastern European and Central Asian countries [1-3]. The risk of hepatitis due to vertical transmission or from exposure in early childhood (e.g., medical exposure to contaminated equipment, household transmission, child-to-child transmission) is of particular concern because infection at a young age is associated with a substantial increased risk

\footnotetext{
* Correspondence: maiabutsashvili@gmail.com

${ }^{1}$ National Center for Disease Control and Public Health (NCDC), 9 Asatiani st., Tbilisi 0177, Georgia

Full list of author information is available at the end of the article
}

of liver disease (e.g., cirrhosis, hepatocellular carcinoma) later in life $[4,5]$.

Fortunately, HBV is preventable through hepatitis B vaccination. The vaccine has been shown to be safe and efficacious for newborns and children [6,7]. Countries which have implemented universal newborn HBV vaccination have reported substantial declines in HBV incidence that is associated with these increases in vaccination coverage $[8,9]$. Thus, most cases of $\mathrm{HBV}$ and its sequelae could be prevented by a universal newborn HBV vaccination program. The success of such public health initiatives depends largely on the degree to which health care providers promote vaccination.

Despite the availability of free vaccine for newborns in Georgia, anecdotal information suggested that health

\section{Biomed Central}


care worker $(\mathrm{HCW})$ concerns about the safety of the HBV vaccine posed an important challenge to universal vaccine coverage for newborns [10]. The study conducted among 297 HCWs reported that only 54\% of study participants would recommend the vaccine to other HCWs. Perception of vaccine safety was identified as the most important predictor for acceptance (Prevalence Ratio (PR) $=3.25$ [95\% CI:1.19, 8.90]) and for willingness to recommend $\mathrm{HBV}$ vaccine to their colleagues [11].

While neonatologists and pediatricians are responsible for newborn HBV vaccinations, HCWs of other specialties likely have a significant indirect impact on vaccine coverage in the general population. Georgians, like those of every culture, also seek advice for health-related decisions from relatives or friends, some of whom may be physicians and nurses. This form of education may supersede the advice of physicians who are known only through a transient patient-provider relationship. Thus, the casual dialogue about $\mathrm{HBV}$ vaccination shared by HCWs among acquaintances, friends and family, and accordingly, their willingness to advise vaccination, may affect $\mathrm{HBV}$ vaccination coverage among the most vulnerable population-newborns.

The purpose of this study was to identify factors associated with HCWs recommendations for newborn HBV vaccination. The hypothesis was that HCWs who were not vaccinated, and thus had no personal experience with the vaccine, would be more likely to recommend against $\mathrm{HBV}$ vaccination of newborns.

\section{Methods}

\section{Study design and sample}

A cross-sectional study on the prevalence and awareness of HBV and other infections was conducted among randomly selected physicians and nurses employed in seven Georgian hospitals between January 1, 2006 and December 31, 2007. Self-administered questionnaires included a module on recommendations for $\mathrm{HBV}$ vaccine for newborns. These physicians were also underwent serological testing for HBV, HCV and HIV.

Seven major hospitals located in five cities were chosen to be representative of Georgia, including three from Tbilisi, the capital and largest city, two from Western Georgia and two from Eastern Georgia. Study participants were selected using simple random sampling of physicians and nurses of selected departments based on lists provided by the hospitals of all full-time employees. The departments enlisted for the study included internal medicine, obstetrics, surgery, intensive care, dialysis and paediatrics. Enrolment continued until the designated number (proportional to number of HCWs per hospital) was met.

\section{Enrollment and data collection}

Potential study participants were invited to voluntarily participate during a private interview with a researcher. The study, including the content of the questionnaire and testing for HBV, HCV and HIV were explained and informed consent was obtained. To maintain confidentiality, a study code was provided to each $\mathrm{HCW}$ and the code was used to label questionnaires and blood samples. Furthermore, a study physician provided test results to all participants along with counselling and referral when further tests were needed. Approval by both Georgian and US Ethics Committees was obtained prior to initiation of the study.

The questionnaire was pilot tested in a small group of physicians and nurses and modified prior to the study. Formative evaluation of the questionnaire was conducted using qualitative methods to assess clarity of the questions. The questionnaire included demographic, clinical and occupational characteristics and knowledge, attitudes, and practices requiring exposure to, and protection from, blood-borne pathogens. The HCWs' willingness to recommend $\mathrm{HBV}$ vaccine to newborns was assessed by the question: "Would you advise HBV vaccine for newborns?" Factors hypothesized to be associated with recommending HBV vaccine for newborns were included in the analyses, including self-reported history of HBV vaccination, fear of vaccine-related side effects, misconceptions of vaccine effectiveness, demographic characteristics (e.g., age, gender), clinical characteristics (e.g., immune status to HBV and HCV) and occupational characteristics (e.g., job category), position (e.g., physicians, nurses), and risky occupational behaviour (e.g., accidental needle-sticks and blood splashes).

\section{Laboratory analysis}

Venous blood $(2-3 \mathrm{ml})$ was drawn from health care workers and transported daily from the hospitals to the laboratory in Tbilisi for storage and analysis. To determine HBV infection status, third generation ELISA tests (manufacturer Orgenics, Israel) were used for HBsAg and anti-HBc. All HBsAg-positive samples were further investigated by confirmatory assays, with immunoreaction of neutralization combined with HBsAg.

\section{Statistical analysis}

Data were checked for quality, double-entered into SPSS (Version 13.0) and verified, before analysis using SPSS and SAS (Version 9.2). Bivariate analyses were performed to assess the association between a history of being vaccinated and each potential predictive factor as well as willingness to advise vaccination for newborns. Multivariate analysis was conducted using Poisson regression with robust variance estimators to identify the 
variable most predictive of recommending $\mathrm{HBV}$ vaccine to newborns.

\section{Results}

Of the 1600 randomly selected HCWs, 1386 (86\%) enrolled in the study and $1328(83 \%)$ provided sufficient information for inclusion in the analysis (Answered the question: Would you recommend HBV vaccine to children?). Of these $1328 \mathrm{HCWs}, 22.80 \%$ were 35 years of age or younger; $20.86 \%$ were males. The study participants were from a broad range of specialties, including surgery $(28.60 \%)$, internal medicine $(19.58 \%)$, intensive care (18.45\%), obstetrics-gynaecologists (15.44\%), dialysis (6.93\%), paediatricians (6.63\%) and others (4.14\%). $46.76 \%$ of respondents were physicians, $28.40 \%$ were from the age group of 35 or younger. $387 \mathrm{HCWs}$ (29.14\%) had $\mathrm{HBc}$ antibodies and 26 (1.96\%) were HBsAg positive.

Overall, 478 (36\%) study participants reported recommending against $\mathrm{HBV}$ vaccination for children. Of the 279 who reported a specific reason, the most common was concern about an adverse vaccine event (38\%, 111/ 279 ) followed by belief that the vaccine was not effective (23\%, 64/279).

Based on the bivariate analysis, the strongest correlate for likelihood of recommending HBV vaccination was the HCW's own vaccination status. Unvaccinated HCWs were much more likely to recommend against HBV vaccine than their vaccinated colleagues $(40.40 \%$ vs $11.40 \%$, respectively, prevalence ratio $(\mathrm{PR})=3.54 ; 95 \% \mathrm{CI}: 2.38$, 5.29). Age was also a factor, with older HCWs more likely to recommend against $\mathrm{HBV}$ vaccine compared to those younger than age $35(39.20 \%$ vs $28.40 \%$, respectively). There was no substantive difference between the recommendations for physicians compared to nurses. A third of paediatricians recommended against HBV vaccine, similar to other HCWs. Dialysis HCWs were the most likely to recommend vaccination, with only $10.9 \%$ $(\mathrm{PR}=3.03$; 95\% CI: 1.58, 5.85) recommending against it. By multivariate analysis, the only significant predictor of recommending HBV vaccine for newborns was the HCW's own vaccination status (Table 1).

\section{Factors related to vaccination status}

Age group was significantly associated with vaccination status: HCWs younger than 35 were more likely to be vaccinated compared to older colleagues $(22.80 \%$ vs $12.30 \%, P R=1.85$; $95 \%$ CI:1.42, 2.43). There was no statistically significant difference in the prevalence of anti$\mathrm{HBC}$ in vaccinated and unvaccinated persons $(28.10 \%$ anti-HBc positives among vaccinated versus $29.40 \%$ among unvaccinated $\mathrm{HCWs}$ ). In the subgroup of HCWs 35 years or younger, the prevalence of anti-HBc was $20 \%$ among vaccinated persons as compared to $24.20 \%$ among unvaccinated HCWs (difference not significant). Gender, occupation and history of occupational exposure to patient's blood (blood splash, needle-stick, cut with contaminated instrument) were not associated with HBV vaccination.

\section{Discussion}

As in other countries $[10,12]$, concerns about vaccine safety persist in Georgia among the general population as well as among HCWs. What may be unique to this country is a pervasive and persistent concern that transcends the safety data for this vaccine. While HBV vaccine has an excellent safety and efficacy record [13-17], perceptions in Georgia have been affected by general concerns about vaccine safety and effectiveness and specifically by one highly publicized neurologic event that occurred a week after an HBV vaccination in Georgia [10]. Overcoming these misconceptions to develop a strong and sustainable universal vaccination program is crucial to reduce HBV in newborns and children and help eliminate HBV- associated liver disease and the economic burden of this disease for Georgia.

This study found that $36 \%$ of HCWs did not recommend HBV vaccination for newborns. Only two groups of HCWs appeared to be importantly different: dialysis workers who see the impact of HBV among their patients at very high risk of the infection, and HCWs who are vaccinated against HBV. However, as evidence of the intractable nature of vaccine safety concerns, one in every ten of these HCWs would also recommend against HBV vaccine for newborns. Age correlated with vaccine status, with older HCWs being the least likely to be vaccinated and least likely to recommend vaccine. These findings provide insight into the demographics of concern about vaccine safety and inform the development of potential interventions to improve knowledge about $\mathrm{HBV}$ vaccination.

There was no statistically significant difference in the prevalence of anti-HBc in vaccinated and unvaccinated persons. This could be probably explained by the fact that during the HCW's HBV vaccination campaign in 2001 there was no HBV antibody testing conducted prior to $\mathrm{HBV}$ vaccination and those being already HBV infected without knowing it received the vaccine.

During the study period over $80 \%$ of $\mathrm{HCW}$ s were not yet vaccinated against HBV. While this finding is similar to many developing countries [18-20], it is the polar opposite of most developed countries where the majority of HCWs are vaccinated [21-24]. These unvaccinated HCWs are substantially less likely to recommend vaccine to newborns. Fear of adverse events and lack of knowledge regarding vaccine efficacy are major factors affecting many HCWs recommendations and personal health practices. While these issues may be addressed by 
Table 1 Bivariate and multivariate analyses from HCW survey: factors related to not recommending HBV vaccine for children, Georgia, 2006-2007

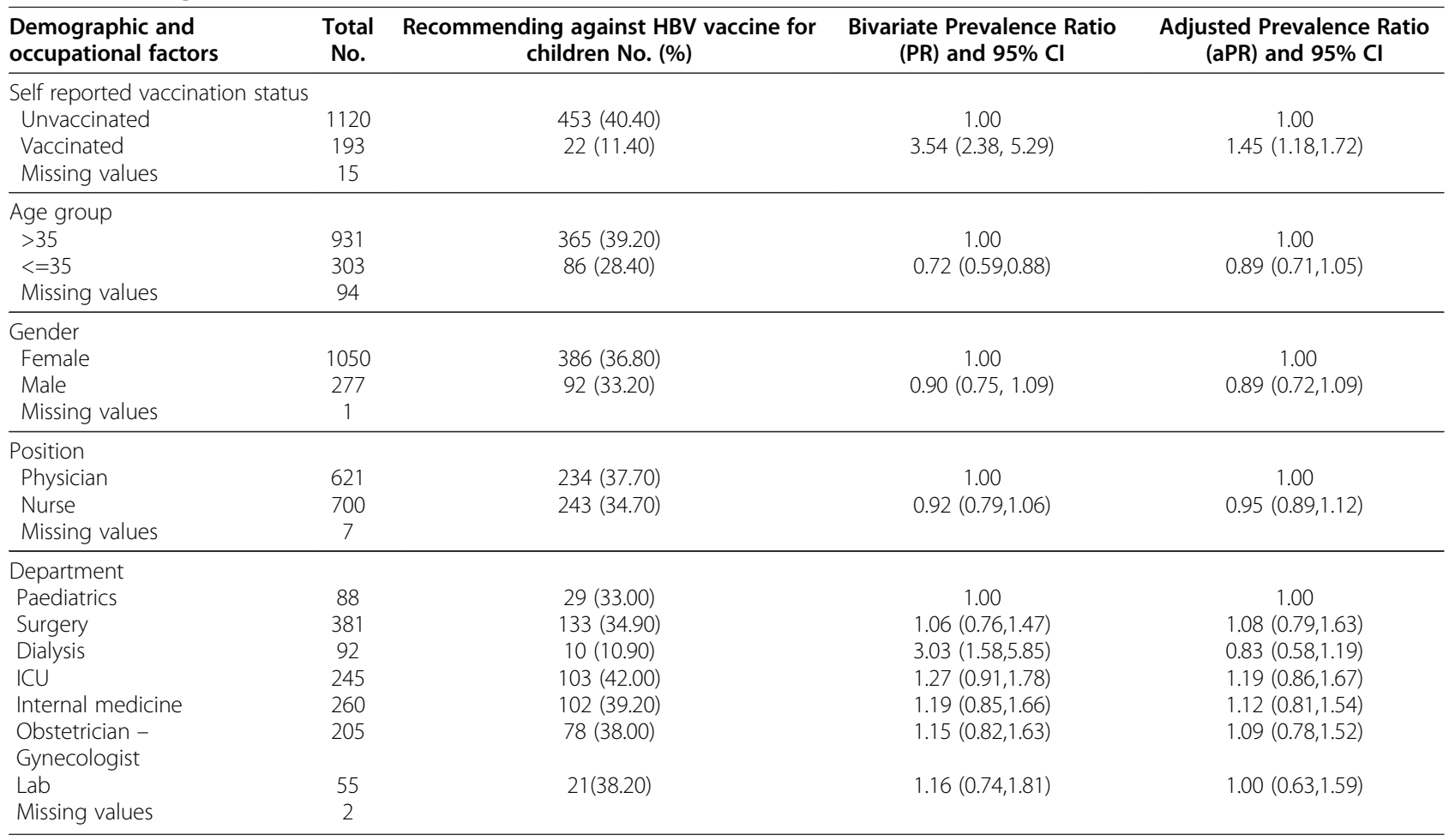

educational programs, the specific experiences of this population suggest educational programs alone are insufficient. Targeted programs which specifically address the publicized event and shed light on the facts may help assuage some concerns.

Personal experience with HBV vaccine appears to be associated with recommending vaccine to newborns.

Having received $\mathrm{HBV}$ vaccine is associated with a healthcare worker recommending the vaccine to newborns. The reasons a healthcare worker might receive the vaccine may be complex. Yet access to the vaccine may be a key factor. If the vaccine is available to the HCW and the barrier of cost is removed, it seems likely that the HCW may consider the personal cost-benefit relationship in a different context. They ultimately may see the benefits of the vaccine as more favourable, receive the vaccine and ultimately be more likely to recommend HBV vaccination for newborns as well as others.

Vaccination programs for HCWs can be justified based on their occupational risk of infection. Cost is always a factor in any vaccine initiative. Some hospitals in a neighbouring country, Turkey, have screened HCWs upon employment and identified those susceptible to HBV for vaccination. This screening and vaccine program is cost-effective given the relatively large proportion of HCWs that have already been exposed to $\mathrm{HBV}$. In Georgia, approximately $30 \%$ of HCWs have evidence of prior HBV exposure and thus would not directly benefit from the vaccine. The remaining HCWs would benefit from the vaccine and safety data suggests that those HCWs who were already vaccinated would not be harmed by revaccination (essentially an additional booster) $[25,26]$. This last point is important because one significant barrier to a screening program is the potential harm caused by employers identifying HCWs who are infectious. Thus, creating an accessible national program for $\mathrm{HCW}$ vaccination must rely on a confidential screening program, or universal vaccination for those with an unreliable vaccination history.

Cost-effectiveness studies on HBV vaccine programs for HCWs typically focus on the occupational risks of HBV infection and benefit of the vaccine to protect HCWs and patients $[27,28]$. This study suggests increasing the proportion of HCWs vaccinated for HBV may increase vaccination coverage of newborns and children.

This study had a strong sampling design and benefited from laboratory testing to establish exposure to HBV. Like all studies, it also had limitations. The primary limitation is that all other information was self-reported. Based on the results, there does not appear to be substantial bias due to social desirability as the proportion of HCWs recommending vaccine is relatively low. Another limitation is that participation was not $100 \%$. It is 
possible that HCWs with active disease were more likely to refuse participation. However, this would not alter the overall conclusions.

The prevalence of HBV infection (anti-HBc) among study participants was about $29 \%$, higher than the prevalence among the general population in Georgia (20\%) [29]. This difference could be explained by the occupational exposure to HBV among HCWs. Even if the majority of HCWs are not likely to develop significant liver disease sequelae if infected later in life, they can transmit the virus to patients and household members who have not been vaccinated. Thus, it would be useful to assess cost-effectiveness of screening for both HBV antibodies prior to vaccinating susceptibles and universal vaccination for HCWs in the absence of screening. In either case, cost-effectiveness analyses of HBV vaccination programs for HCWs should broaden their assessment of benefits.

In 2009 the Ministry of Labour, Health, and Social Affairs of Georgia and the National Center for Diseases Control established a hepatitis B catch-up vaccination program for at-risk healthcare workers, medical students, and adolescents in three regions of the country: Tbilisi (capital city with $1 / 3$ of Georgia population), Imereti and Adjara (regions in western Georgia) with support of Rostropovich-Vishnevskaya Foundation.

By 2010, 12,963 health care workers have been screened for hepatitis B (Anti-HBc) and 4,579 (35.3\%) appeared to be $\operatorname{Anti}-\mathrm{HBC}(+)$. The rest of $\mathrm{HCWs}$ were offered administration of HBV vaccine on a voluntary basis, from which 7,000 have been vaccinated against hepatitis B.

No study was conducted to estimate the impact of vaccination campaign among HCWs on the attitude of $\mathrm{HCW}$ towards $\mathrm{HBV}$ vaccine and their willingness to recommend it to the children.

\section{Conclusion}

Our study reinforced the importance of HBV vaccination among HCWs for the obvious direct occupational benefits such protection confers as well as possibly recommending vaccination for newborns. This study leads us to recommend the education and outreach targeted towards HCWs who are least likely to perceive the benefits of $\mathrm{HBV}$ vaccination; those over age 35, may improve the long-term HBV vaccine prevalence rates. Such programs must also remove barriers to vaccination such as concern for social consequences and personal financial burden.

\section{Competing interests}

The authors declare that they have no competing interests.

\section{Authors' contributions}

$\mathrm{MB}$ - Pl, development of study design, analysis of data, and manuscript writing. GK - Manager, overall management of the study and participation in manuscript writing. MT - Epidemiologist, participation in data collection and analysis. DM- Participation in development of the study design and editing the manuscript. WT- Participation in development of research tools and editing the manuscript. JD- Participation in drafting and editing the manuscript. KN- editing the manuscript. LAM - US Co-PI, collaborative overall supervision of the study, manuscript writing. All authors read and approved the final manuscript.

\section{Acknowledgements}

This project was supported by Civilian Research and Development Foundation (CRDF) grant \# GEB2-2636-TB-05 and NIH Research Grants \# 2D43TW000233 and 5D43TW007384 funded by the Fogarty International Center. All authors report no conflicts of interest relevant to this study.

\section{Author details}

${ }^{1}$ National Center for Disease Control and Public Health (NCDC), 9 Asatiani st., Tbilisi 0177, Georgia. ${ }^{2}$ Maternal and Child Care Union, Tbilisi, Georgia. ${ }^{3}$ Division of Foodborne, Waterborne and Environmental Diseases at CDC, Atlanta, GA, USA. ${ }^{4}$ Department of Emergency Medicine, at Albany Medical College, Albany, NY, USA. ${ }^{5}$ Department of Medicine, AT SUNY-Downstate Medical Center, Brooklyn, NY, USA. ${ }^{6}$ Johns Hopkins Bloomberg School of Public Health, Baltimore, MD, USA. ${ }^{7}$ University at Albany, School of Public Health, Rensselaer, NY, USA.

Received: 20 January 2012 Accepted: 14 December 2012 Published: 20 December 2012

\section{References}

1. Butsashvili M, Tsertsvadze T, McNutt LA, et al: Prevalence of hepatitis B, hepatitis C, syphilis and HIV in Georgian blood donors. Eur J Epidemiol 2001, 17(7):693-695.

2. Iashina TL, Favorov MO, Shakhgil'dian IV, Firsova SN, Eraliev AE, Zhukova LD, Reznichenko RG: The prevalence of the markers of viral hepatitis $B$ and delta among the population in regions differing in the level of morbidity. Vopr Virusol 1992, 37(4):194-196.

3. Nurgalieva ZZ, Hollinger FB, Graham DY, Zhangabylova S, Zhangabylov A: Epidemiology and transmission of hepatitis $B$ and $C$ viruses in Kazakhstan. World J Gastroenterol 2007, 13(8):1204-1207.

4. Zacharakis G, Koskinas J, Kotsiou S, Pouliou E, Papoutselis M, Tzara F, Vafeiadis N, Maltezos E, Archimandritis A, Papoutselis K: Natural history of chronic hepatitis $B$ virus infection in children of different ethnic origins: a cohort study with up to 12 years' follow-up in northern Greece. J Pediatr Gastroenterol Nutr 2007, 44(1):84-91.

5. Slowik MK, Jhaveri R: Hepatitis $B$ and $C$ viruses in infants and young children. Semin Pediatr Infect Dis 2005, 16(4):296-305.

6. Lewis $E$, Shinefield HR, Woodruff BA, et al: Safety of neonatal hepatitis B vaccine administration. Pediatr Infect Dis J 2001 20:1049-1054

7. Tovo PA, Lazier L, Versace A: Hepatitis $B$ virus and hepatitis $C$ virus infections in children. Curr Opin Infect Dis 2005, 18(3):261-266.

8. Van Herck K, Van Damme P: Benefits of early hepatitis B immunization programs for newborns and infants. Pediatr Infect Dis J 2008, 27(10):861-869.

9. Papaevangelou V, Hadjichristodoulou C, Cassimos DC, Pantelaki K, Tzivaras A, Hatzimichael A, Theodoridou M: Seroepidemiology of hepatitis B in Greek children 6 years after the implementation of universal vaccination. Infection 2008, 36(2):135-139.

10. Case report: encephalomyelitis caused by hepatitis B vaccination. 2007. Available at: http://www.vaclib.org/basic/Case_Report.doc. Accessed: April 29.

11. Topuridze M, ButsaShvili M, Kamkamidze G, et al: Hepatitis B vaccine coverage among health care workers: barriers to coverage. Infect Control Hosp Epidemiology 2010, 31(2):158-164.

12. Balinska MA: Hepatitis $B$ vaccination and French Society ten years after the suspension of the vaccination campaign: how should we raise infant immunization coverage rates? Review. J Clin Virol 2009, 46(3):202-205. Review. 
13. Smith PJ, Kennedy AM, Wooten K, Gust DA, Pickering LK: Association between health care providers' influence on parents who have concerns about vaccine safety and vaccination coverage. Pediatrics 2006, 118(5):e1287-e1292.

14. Alavian SM, Mansouri S, Abouzari M, Assari S, Bonab MS, Miri SM: Long-term efficacy of hepatitis B vaccination in healthcare workers of Oil company hospital, Tehran, Iran (1989-2005). Eur J Gastroenterol Hepatol 2008, 20(2):131-134.

15. Szmuness W, Stevens $C E$, Harley EJ, Zang EA, Alter HJ, Taylor PE, et al: Hepatitis B vaccine in medical staff of hemodialysis units: efficacy and subtype cross-protection. N Engl J Med 1982, 307:1481-1486.

16. Mac Mahon BJ, Helminiak C, Wainwright RB, Bulkow L, Wainwright K: Frequency of adverse reactions to hepatitis $B$ vaccine in 43,618 persons. Am J Med 1992, 92:254-256.

17. Zanetti AR: Update on hepatitis B vaccination in Italy 10 years after its implementation. Vaccine 2001, 19:2380-2383.

18. Braka F, Nanyunja M, Makumbi I, Mbabazi W, Kasasa S, Lewis RF: Hepatitis B infection among health workers in Uganda: evidence of the need for health worker protection. Vaccine 2006, 24(47-48):6930-6937.

19. Suckling RM, Taegtmeyer M, Nguku PM, Al-Abri SS, Kibaru J, Chakaya JM, Tukei PM, Gilks CF: Susceptibility of healthcare workers in Kenya to hepatitis $B$ : new strategies for facilitating vaccination uptake. J Hosp Infect 2006, 64(3):271-277.

20. Talaat M, Kandeel A, El-Shoubary W, Bodenschatz C, Khairy I, Oun S, Mahoney FJ: Occupational exposure to needlestick injuries and hepatitis $B$ vaccination coverage among health care workers in Egypt. Am J Infect Control 2003, 31(8):469-474.

21. Simard EP, Miller JT, George PA, Wasley A, Alter MJ, Bell BP, Finelli L: Hepatitis $B$ vaccination coverage levels among healthcare workers in the United States, 2002-2003. Infect Control Hosp Epidemiol 2007, 28(7):783-790.

22. Batista SMF, Andreas MSA, Borges AMT, Lindenberg ASC, Silva AL, Fernandes TD, Pereira EF, Basmage EAM, Cardoso DDP: Seropositivity for hepatitis $B$ virus, vaccination coverage, and vaccine response in dentists from Campo Grande, Mato Grosso do Sul, Brazil. Mem Inst Oswaldo Cruz 2006, 101(3):263-267.

23. Dannetun E, Tegnell A, Torner A, Giesecke J: Coverage of hepatitis B vaccination in Swedish healthcare workers. J Hosp Infect 2006, 63(2):201-204.

24. Vranckx R, Jacque P, De Schrijver A, Moens G: Hepatitis B vaccination coverage in Belgian health care workers. Infection 2004, 32(5):278-281.

25. Chen W, Gluud C: Vaccines for preventing hepatitis B in health-care workers. Cochrane Database Syst Rev 2005, 19(4):CD000100.

26. Floreani A, Baldo V, Cristofoletti M, Renzulli G, Valeri A, Zanetti C, Trivello R: Long term persistence of anti-HBs after vaccination against HBV: an 18 year experience in health care workers. Vaccine 2004, 22(5-6):607-610

27. Kuruuzum Z, Yapar N, Avkan-Oguz V, Aslan H, Ozbek OA, Cakir N, Yuce A: Risk of infection in health care workers following occupational exposure to a noninfectious or unknown source. Am J Infect Control 2008, 36(10):e27-e31.

28. Tu HA, Woerdenbag HJ, Kane S, Riewpaiboon A, van Hulst M, Postma M: Economic evaluations of hepatitis $B$ vaccination for developing countries. Expert Rev Vaccines 2009, 8(7):907-920.

29. National Center Of Disease Control And Public Health (Ncdc), Bulletin. http://www.ncdc.ge/GEO/Publications/Bulletin/ bulletin_2005/Bul_1.pdf.

doi:10.1186/1471-2334-12-362

Cite this article as: Butsashvili et al:: Associated factors for recommending HBV vaccination to children among Georgian health care workers. BMC Infectious Diseases 2012 12:362.

\section{Submit your next manuscript to BioMed Central and take full advantage of:}

- Convenient online submission

- Thorough peer review

- No space constraints or color figure charges

- Immediate publication on acceptance

- Inclusion in PubMed, CAS, Scopus and Google Scholar

- Research which is freely available for redistribution

Submit your manuscript at www.biomedcentral.com/submit
() Biomed Central 